\title{
Analysis of light curve and orbital period of contact binary $\mathrm{NO} \mathrm{Vul}$
}

\author{
Muhammed Faruk YILDIRIM ${ }^{1,2 *}$, Faruk SOYDUGAN ${ }^{1,2}$ \\ ${ }^{1}$ Art and Science Faculty, Department of Physics, Çanakkale Onsekiz Mart University, Çanakkale, \\ Turkey. \\ ${ }^{2}$ Astrophysics Research Center and Ulupınar Observatory, Çanakkale Onsekiz Mart University, \\ Çanakkale, Turkey.
}

Gelis Tarihi (Received Date): 18.04.2020

Kabul Tarihi (Accepted Date): 09.06.2020

\begin{abstract}
In this study, we investigated light curve analysis of contact binary system NO Vul. Also, we investigated orbital period variations of contact binaries NO Vul based on all published minima times and also those obtained from our own observations. Photometric observations are obtained from the ASAS public archive. Physical parameters of the system are derived using the modeling code by Wilson and Dewinney's (WD). Photometric results indicate that NO Vul is $q=0.72(2)$ and about $f=\% 19$ respectively and the derived orbital inclination $i=77(1)$.
\end{abstract}

Keywords: Contact binary stars, light curve anaysis, orbital period analysis, NO Vul.

\section{Değen çift NO Vul'un 1şık eğrisi ve yörünge dönem analizi}

$\ddot{\mathbf{O} z}$

Bu çalışmada, NO Vul değen sistemin lşık ĕgrisi analizi yapılmıştır. Bununla birlikte NO Vul değen sisteminin yörünge dönem değişimi, literatürde yayınlanan minimumlar ve bu çalışma kapsamında yapılan gözlemlerden elde edilen minimumlarla yapılmıştır. Sistemin ışık eğrisi analizi için ASAS veri arşivinden faydalanılmıştır. Sistemin ışık eğrisi analizi Wilson \& Dewinney yazılımı kullanılarak yapılmıştır. Fotometrik sonuçlardan, sistemin kütle oranı (q) 0.72(2), değme/taşma parametresi (f) yaklaşık \%19 ve yörünge eğikliği (i) 77(1) olarak belirlenmiştir.

\footnotetext{
* Muhammed Faruk YILDIRIM, mf.yildirim@hotmail.com.tr, http://orcid.org/0000-0003-2382-7011

Faruk SOYDUGAN, fsoydugan@ @omu.edu.tr, http://orcid.org/0000-0002-5141-7645
} 
Anahtar kelimeler: Değen çift yıldızlar, ışık ĕgrisi analizi, yörünge dönemi analizi, NO Vul.

\section{Introduction}

Investigation of contact and overcontact binaries, according to the appearance of their light curves, is important to explore the evolutional properties of the binary stars. Depths of the primary and secondary minimum in the light curves of mostly contact binaries are approximately the same. In such systems, the surface temperatures of the component stars are almost the same. The orbital periods of W UMa type eclipsing binary systems are usually short and are between 0,2 and 0,8 days. Mass ratios $\left(q=M_{2} / M_{1}\right)$ of the systems are mostly between $0,1-0,5$. Spectral types of such systems vary from late A to middle $\mathrm{K}$. Evolutionary status of interacting binaries around the contact status can be mostly clarified by the thermal relaxation oscillation (TRO) theory [1]. A binary system in thermal contact demonstrates the light curves like those of W UMa type binaries with nearly equal depths. On the other hand, those in broken contact phase have the components with different temperatures. In this study, the V-light curve and orbital period change of a contact binary NO Vul has been analyzed and discussed.

NO Vul (2MASS J19343799+2037142, V=12 ${ }^{\mathrm{m}} .83$ ) is a contact binary system. The eclipsing binary system was discovered by [2]. Analysis of the period variations of the system was made by [3]. [4] were solved out R-light curve and made orbital period analysis of the system. Recently, period variation of the system was carried out by [5]. Since all the published minima times of the system are in the O-C gateway, they have not given as reference. There is no radial velocity study in the literature of the contact binary NO Vul. Therefore, the mass ratio of the system has not been determined. This is why q search of the target system was done in this study (see Fig. 1.). Table 1, provides basic information about the NO Vul system.

Table 1. Basic information of NO Vul components (The figures on the basic physical parameters represent references).

\begin{tabular}{cccccccc}
\hline \multirow{2}{*}{ System } & Spectral & Period & To & $M_{1}$ & $R_{1}$ & & \\
& Type & (day) & (HJD) & $\left(M_{\odot}\right)$ & $R_{2}$ & $a$ & References \\
& $\left(R_{\odot}\right)$ & $\left(R_{\odot}\right)$ & \\
\hline NO Vul & F8+F8.5 & $0.37076621^{7}$ & $2446346.2933^{7}$ & $1.33^{6}$ & $1.24^{6}$ & $2.66^{6}$ & {$[6,7]$} \\
& & & & $0.52^{6}$ & $0.70^{6}$ & & \\
\hline
\end{tabular}

\section{Material and method}

\subsection{Photometric observations}

NO Vul's observations were made at Çanakkkale Onsekiz Mart University Ulupınar Observatory. Observations LX200 model Cassegrain-Schmidt type, 305mm Apogeee Alta U47 CCD was made using a diameter telescope and a 15',8 $\times 15^{\prime}, 8$ viewing angle detector. The observations were made in total three nights in May (2013), July (2012) and August (2012) of 2012 and 2013 years. During the observations, as comparison and check stars, respectively; GSC 1609-1367 and GSC 1609-1497 stars are selected. During the observations, no significant change was observed in the light changes of the comparison and check stars. The observational errors were measured as approximately 0.01 magnitude in all filters. The reduction was carried out using the C-Munipack program 
(http://cmunipack.sourceforge.net). Also from our observations, three minima (see Table 2) times were determined for $\mathrm{NO}$ Vul.

Table 2. New minima times obtained in this study for NO Vul.

\begin{tabular}{cccc}
\hline Filter & $\begin{array}{c}\text { Minima Times } \\
(\mathrm{HJD}+2400000)\end{array}$ & Errors & $\begin{array}{c}\text { Type } \\
(\mathrm{I} / \mathrm{II})\end{array}$ \\
\hline$B V R$ & 56133.3900 & 0.001 & $\mathrm{I}$ \\
$B V R$ & 56170.4670 & 0.002 & $\mathrm{I}$ \\
$B V R$ & 56433.5253 & 0.002 & II \\
\hline
\end{tabular}

\subsection{Analysis of V-light curve}

In order to analyze light curve of $\mathrm{NO}$ Vul, we have preferred the Wilson-Devinney code, which is the well-known and used for the light and radial curve analysis of the binary systems [8]. During the analysis, mode 3 of Wilson-Devinney code, which holds for contact systems, is used.

In this study, only light changes were obtained for the minimum time and the entire light curve was not observed, so ASAS data archive was used for the analysis of all light curve. We analyzed V-light curve of the system, which was taken from ASAS public data archive (http://www.astrouw.edu.pl/asas/?page=acvs). In this paper, the data in the ASAS data archive was studied, since it was less scattered and had more good data than other data archives. Effective temperature of $T_{l}=6050 \mathrm{~K}$ was determined from the $B$ - $V$ value (taken from the APASS database (https://www.physics.mcmaster.ca/)) for the system was calculated as 0.56 , while the $6050 \mathrm{~K}$ value was used considering the F9V spectral classification [9] for the primary component. The gravity-darkening coefficients $g_{1}=g_{2}=0.32$ [10] and bolometric albedo values $A_{1}=A_{2}=0.5$ were used, which correspond to the convective envelopes of both components. There is no mass ratio determined in the literature of the system because no radial velocity study has been performed. This is why q search of the target system was done in this study (see Fig. 1.). After determining the best solution as a result of light curve analysis, all values are kept constant. Then the mass ratio parameter is free and the q value is determined. The mass ratio $(q)$ of the system was searched and found to be $q=0.72$ (see Figure 1) in order to use an input parameter for the analysis since there is no published radial velocity measurements of the components. The resulted parameter values obtained in the $\mathrm{V}$-light curve analysis of $\mathrm{NO} \mathrm{Vul}$ are given in Table 3. 


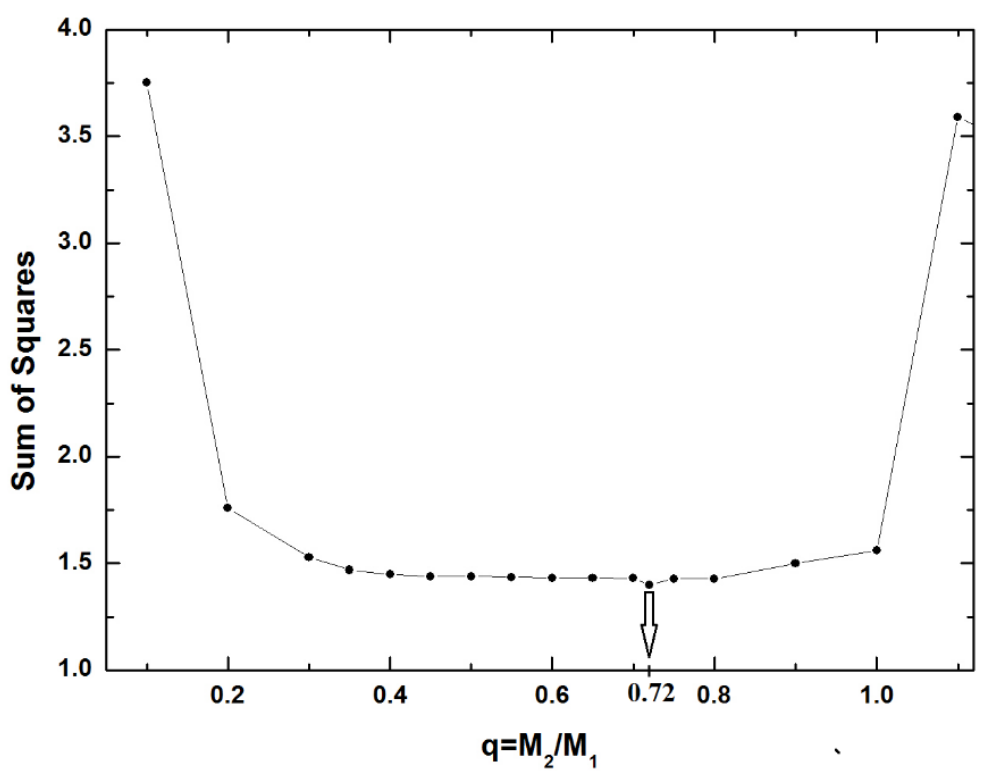

Figure 1. q-search for NO Vul.

Table 3. Final photometric parameters obtained from ASAS V-light curve analysis.

\begin{tabular}{cc}
\hline Parameters & Values and their errors \\
\hline$g_{l}=g_{2}$ & 0.32 \\
$A_{l}=A_{2}$ & 0.5 \\
$i$ (deg.) & $77(1)$ \\
$T_{1}(\mathrm{~K})$ & 6050 \\
$T_{2}(\mathrm{~K})$ & $5545(145)$ \\
$\Omega_{1}=\Omega_{2}$ & $3.201(9)$ \\
$q$ & $0.72(2)$ \\
$\frac{L_{1 v}}{L_{1 v}+L_{2 v}}$ & $0.662(24)$ \\
$\frac{L_{2 v}}{L_{1 v}+L_{2 v}}$ & 0.338 \\
$f(\%)$ & 19 \\
\hline
\end{tabular}

During the photometric analysis, a possible third light was investigated but there was no significant result. As a result of the light curve analysis, the third light contribution was not significant. We continued the iterations until the corrections in the free parameters $(i$ : orbital inclination angle of the system, $T_{2}$ : surface temperature of second component, $q$ : mass ratio, $\Omega_{l}$ : surface potential of the main component and $L_{l}$ : luminosity of the main component) values were small in possible errors. During the analysis, the surface temperature of the first component $\left(T_{1}\right)$, the luminosity of the second component $\left(L_{2}\right)$ and the surface potential of the second component $\left(\Omega_{2}\right)$ have kept constant. A comparison between observed ASAS V-light curve and the best theoretical fits achieved is demonstrated in Figure 2. 


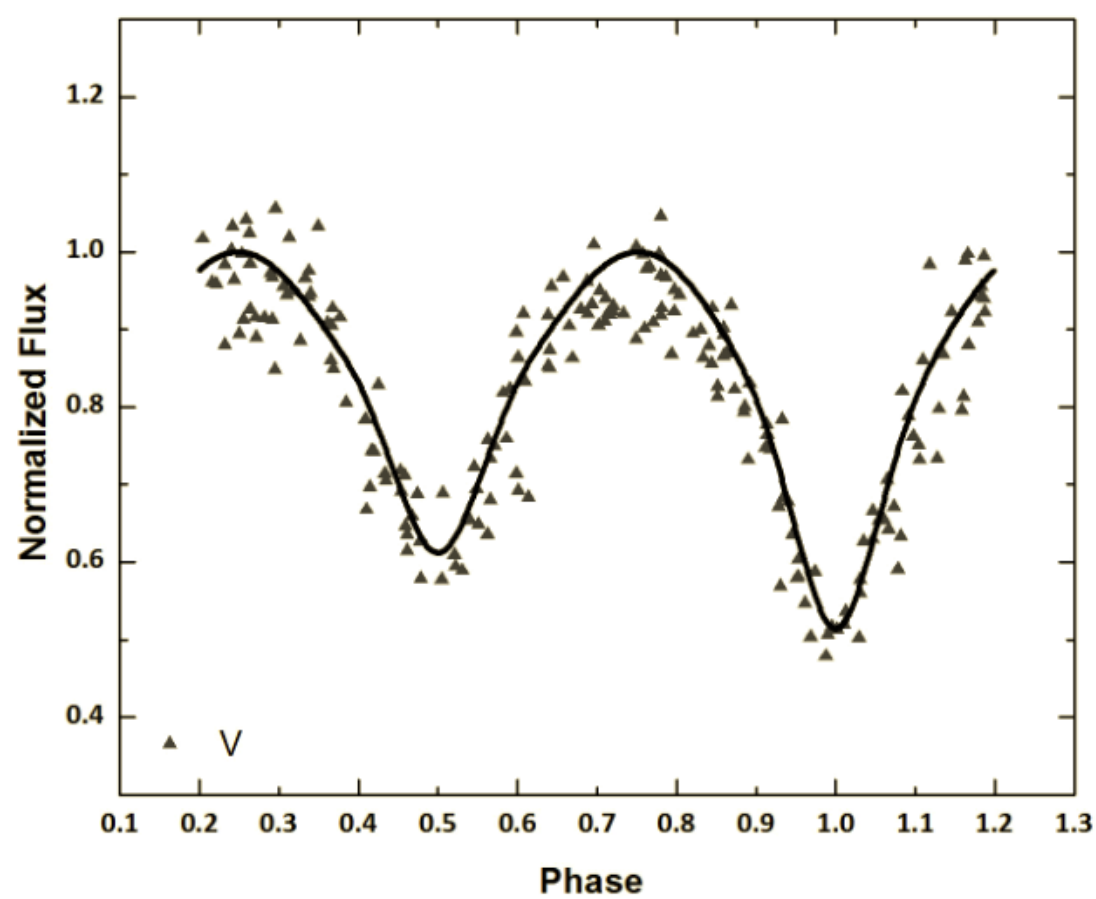

Figure 2. Observed ASAS light curve and calculated theoretical one in V filter (Triangular symbols represent observational data, while the continuous line represents the theoretical fit).

\subsection{Orbital period study}

The presence of variations in the period of a binary stars can be demonstrated by the wellknown $\mathrm{O}-\mathrm{C}$ method. In this method, $\mathrm{O}$ : means the minima observed times and $\mathrm{C}$ : means the calculated minima times. The period change of a system can be easily monitored in the created $\mathrm{O}-\mathrm{C}$ diagram. The distribution in such a diagram can be linear, parabolic, sinusoidal or in a complicated way. In this paper, considering the distributions of O-C data of NO Vul, O-C values are calculated by Equation 1 and Equation 2 given below:

$\operatorname{Min} I=T_{0}+E \cdot P+Q \cdot E^{2}+\Delta t$

$\Delta t=\frac{a_{12} \sin i^{\prime}}{c}\left\{\frac{1-e^{\prime 2}}{1+e^{\prime} \cos v^{\prime}} \sin \left(v^{\prime}+w^{\prime}\right)+e^{\prime} \cos w^{\prime}\right\}$

In the equation $1, T_{0}$ and $P$ represent the initial light elements, $E$ and $Q$ represent the number of cycles and the quadratic term, respectively and $\Delta t$ is the time delay. In the equation 2, $c$ : expresses the speed of light, $a_{12}$ : semi-major axis length of the third possible component, $i$ : orbital inclination, $e$ : orbital eccentricity, $v$ : true anomaly and $w$ : the longitude value of the periastron of the third-body orbit. The description of this equation was given by [11]. The O-C method was used to examine the period change of NO Vul. We have collected all published minima times in the literature for NO Vul binary systems.

In the $O-C$ graph of NO Vul, cyclical change was seen in addition to parabolic behavior. In O-C analyzes, sinus-like changes may either be due to a possible third component or may be due to magnetic activity. It has been interpreted in detail in the result and conclusions section for this change (sinusoidal exchange) of NO Vul system. Therefore, we used Equation 1 to represent the observational data. The result parameters obtained in $O-C$ analysis are listed in Table 4. Using the quadratic term $(Q)$, we have computed the decreasing rate in the orbital period to be -0.88 s century $^{-1}$. In the $O-C$ graph of NO Vul, 
the representation of both parabolic and cyclic variations is indicated in Figure 4. The periodic variation (see Fig 3) was interpreted by an unseen component around the eclipsing pair.

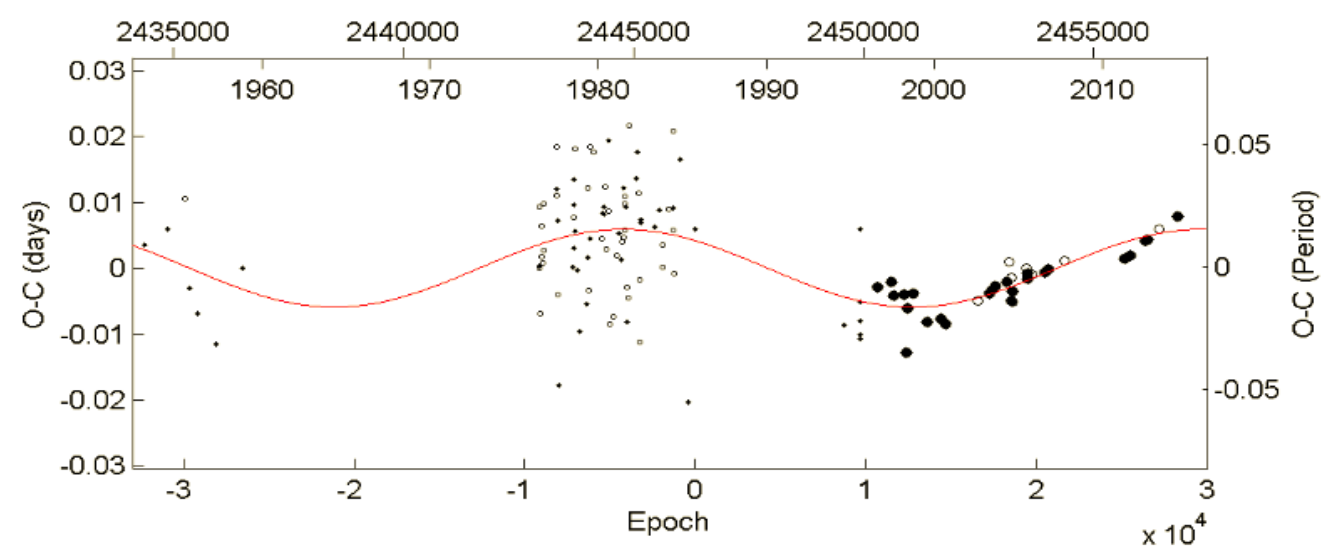

Figure 3. Distribution of $O-C$ data of NO Vul after subtraction of parabola and its theoretical representation by third-body effect.

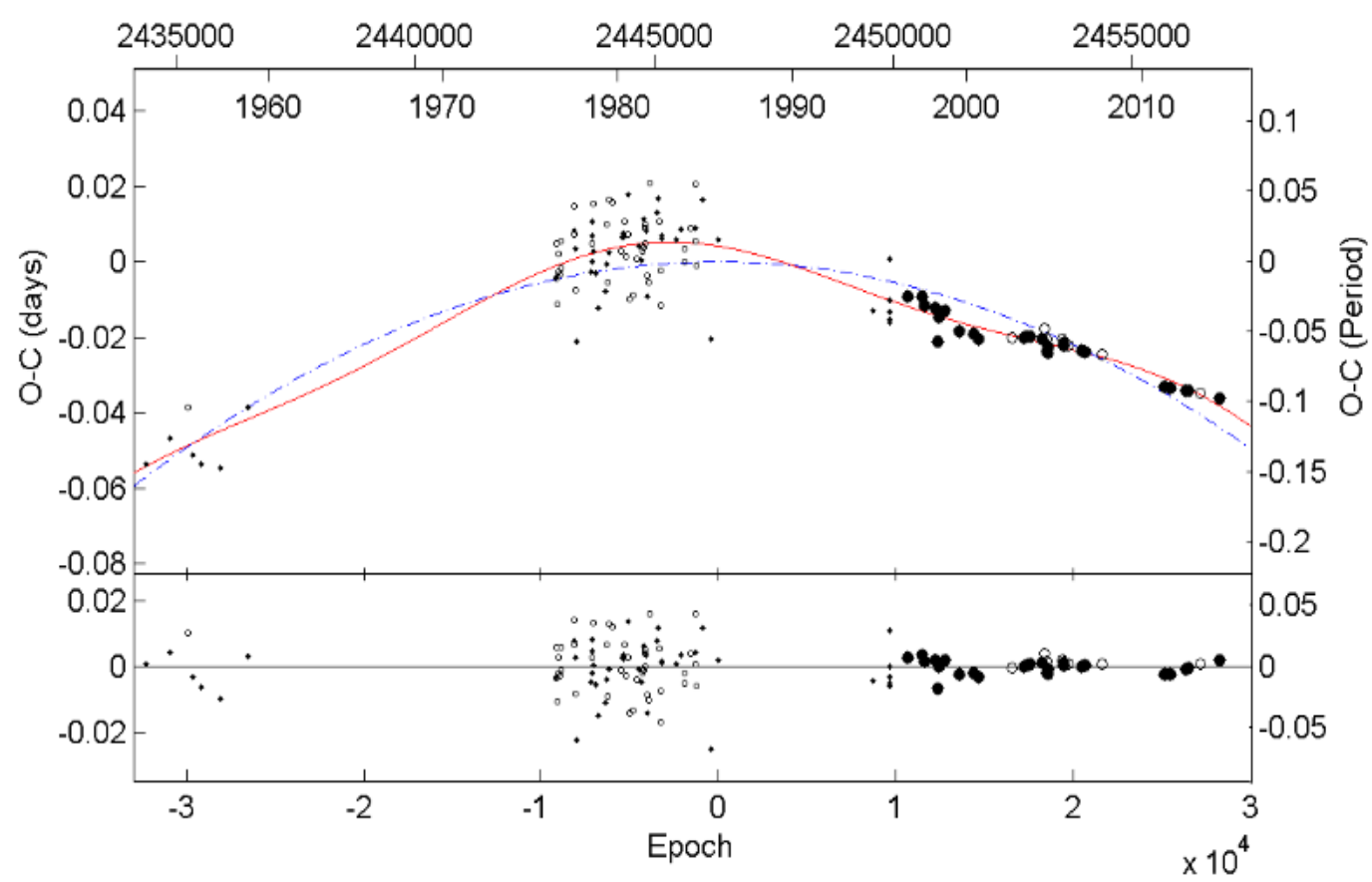

Figure 4. O-C graph for NO Vul, both parabolic (blue line) and sinusoidal exchange (red line) representation. 
Table 4. Resulted parameters from $O-C$ analysis.

\begin{tabular}{cc}
\hline Parameters & NO Vul \\
\hline$T_{\mathrm{o}}(\mathrm{HJD}+2400000)$ & $46346.3010(2)$ \\
$P_{\text {orb }}$ (day) & $0.3707665(1)$ \\
$Q$ (day) & $-5.15(1) \times 10^{-11}$ \\
$\mathrm{~d} P / \mathrm{d} t$ (s/century) & -0.88 \\
$\mathrm{~d} M / \mathrm{d} t(\mathrm{M} \odot /$ year $)$ & $-7.79 \times 10^{-8}$ \\
$a_{12} \sin i(\mathrm{AB})$ & $1.02(15)$ \\
$e$ & 0.0 \\
$\omega(\mathrm{deg})$ & 90 \\
$T^{\prime}(\mathrm{HJD}+2400000)$ & $52315(270)$ \\
$P_{12}($ year $)$ & $34(4)$ \\
$f\left(\mathrm{~m}_{3}\right)\left(M_{\odot}\right)$ & $0.0009(1)$ \\
$m_{3}\left(M_{\odot}\right)$ for $i=90 \mathrm{deg}$. & 0.15 \\
\hline
\end{tabular}

\section{Result and conclusions}

This study focused on the ASAS V-light curve analysis and period change of the contact system NO Vul. The $V$-light curve of the system was analysed using Wilson-Devinney code. We obtained the effective temperature of the cooler companion as $T_{2}=5545(145) \mathrm{K}$ and inclination of the orbit $i=77(1)$ degree. The surface gravitational potential values were found to be $\Omega_{1}=\Omega_{2}=3.201(9)$ and the fractional luminosity of the primary component was calculated to be $L_{1} /\left(L_{1}+L_{2}\right)=0.662(24)$. It is shown that $\mathrm{NO} \mathrm{Vul}$ is a contact system with fill-out factor $f=\% 19$ and mass ratio $q=0.72$. The fill-out factor rate is determined by the $f$ parameter made according to Roche geometry and is calculated with the following equation:

$f=\frac{\Omega_{\text {in }}-\Omega}{\Omega_{\text {in }}-\Omega_{\text {out }}}$

In the Eq.3.; The surface potential value $(\Omega)$ of the binary system is the internal critical Roche equipotential $\left(\Omega_{\text {in }}\right)$ and the external critical Roche equipotential $\left(\Omega_{\text {out }}\right)$.

In this paper, $i, \Omega_{1}=\Omega_{2}$ and $T_{1} / T_{2}$ ratio was found as 77(1), 3.201(9) and 1.09 respectively, while [4] found it as $80.90(0.3), 3.08(0.18), 1.13$ respectively. While we determined the fill-out factor value as 19 percent, [4] determined as 42.9 percent. Since the light curves were scattered in both studies, the results of the studies were different.

Interacting binary NO Vul indicates orbital period decrease with a rate of -0.88 s century ${ }^{-}$ ${ }^{1}$. Conservative mass transfer between components can be recommended as the cause of orbital period decrease. The following equation is used for the calculation of mass transfer value between the components [12].

$\frac{\Delta P}{P}=\frac{3 \dot{m}_{1}\left(m_{1}-m_{2}\right)}{m_{1} \cdot m_{2}}$

where it is represented by the period change $(\Delta P)$ and the transferred mass amount $\left(\dot{m}_{1}\right)$. The mass transfer value from the more massive component to the less massive one is 
calculated to be $-7.79 \times 10^{-8} \mathrm{M} \odot$ for NO Vul. In addition, $O-C$ graph shows cyclic change with a period of about 34 years. Such periodic variations are described in two ways. The first of these, we explained using the light-time effect resulted from a tertiary component around the binary system. The minimum mass of the probable third body was computed as $0.15 \mathrm{M} \odot$.

In this study, the mass function and the orbital period of the hypothetical third body were determined as 0.0009(1) $\mathrm{M} \odot$ and 34(4), while [5] determined as 0.000627(3) M $\odot$, 26.17(5) in their study. In addition, [4] determined the mass of thypothetical third body as $0.36 \mathrm{M} \odot$ in the orbital period analysis study, in our study it was determined as $0.15 \mathrm{M} \odot$. And also in the same study, [4] suggested that the spectral type of the hypothetical third body could be M2.

The second way, in binary stars, if one or both components have the late spectral type (F5 and late type), the probable cause of the cyclic change observed in the $O-C$ distribution may be due to magnetic activity, such a change being explained by the Applegate mechanism [13]. Therefore, we have also calculated Applegate model parameters listed in Table 4 to give an alternative explanation for the cyclic $O-C$ variations. There are no studies showing the magnetic activity in the literature for $\mathrm{NO} \mathrm{Vul} \mathrm{system.} \mathrm{If} \mathrm{the} \mathrm{reason}$ for the cyclical change is magnetic activity, since both components are of the late spectral type from F5, it can be in both components. The applagate parameters calculated for both components appear very close. The Applegate mechanism for cyclical change in any publication in the literature has not been discussed.

Table 5. Applegate parameters for NO Vul.

\begin{tabular}{cc}
\hline Parameters & NO Vul \\
\hline$P_{\text {mod }}($ year $)$ & 34 \\
$\Delta P / P$ & $5,97 \times 10^{-6}$ \\
$\Delta J\left(\mathrm{erg} \mathrm{s}^{-1}\right)$ & $-2,5 \times 10^{47}$ \\
$\Delta \Omega / \Omega$ & 0,001 \\
$\Delta E(\mathrm{erg})$ & $6,4 \times 10^{40}$ \\
$I_{s}\left(\mathrm{~g} \mathrm{~cm}^{2}\right)$ & $9,8 \times 10^{53}$ \\
$\Delta L_{r m s}\left(L_{\odot}\right)$ & 0,05 \\
$B(\mathrm{kG})$ & 8 \\
\hline
\end{tabular}

NO Vul should be also investigated using high-resolution spectra to have more knowledge for its nature. As a result of period analysis of the system, photometric and spectroscopic evidences should be obtained to investigate the mass transfer between components, the presence of a possible third body and the magnetic activity cycle.

\section{Acknowledgment}

The authors thank for the support of the Astrophysics Research Centre and Observatory of Çanakkale Onsekiz Mart University. This research has made use of the APASS database, located at the AAVSO web site. Funding for APASS has been provided by the 
Robert Martin Ayers Sciences Fund. This study made VIZIER and SIMBAD databases at CDS, Strasbourg, France.

\section{References}

[1] Qian, S., Are Overcontact Binaries Undergoing Thermal Relaxation Oscillation With Variable Angular Momentum Loss?, Monthly Notice of the Royal Astronomical Society, 342, 1260-1270 (2003).

[2] Kalv, P. and Leis, L., New eclipsing variables in Vulpecula - SVS 1987 and SVS 1988, Astronomicheskii Tsirkulyar, 793, 8, (1973).

[3] Qian, S. and Ma, Y., Period Studies of Some Neglected Close Binaries: EP Andromedae, V724 Aquilae, SS Comae, AM Eridani, FZ Orionis, BY Pegasi, EQ Tauri, and NO Vulpeculae, The Publications of the Astronomical Society of the Pacific, 113, 754, (2001).

[4] Zasche, P., Wolf, M. and Kotkova, L., The First Light Curve Analysis of Two Overcontact Binaries: EY Cas and NO Vul, Information Bulletin on Variable Stars, 5812, 1 (2008).

[5] Bulut, A., Bulut, I., Çicek C. and Erdem, A., Light-time Effect in two Eclipsing Binaries: NO Vul and EW Lyr, AIP Conference Proceedings, 1815, 1 (2017).

[6] Svechnikov, M.A., Kuznetsova E.F., Catalogue of Approximate Photometric and Absolute Elements of Eclipsing Variable Stars, Ural university publication" (1990).

[7] Kreiner, J.M., Kim, C-H., Nha, II-Seong, An atlas of O-C Diagrams of Eclipsing Binary Stars, Cracow, Poland (2001).

[8] Wilson, R.E. and Devinney, E.J., Realization of Accurate Close-Binary Light Curves: Application to MR Cygni, Astrophysics Journal, 166, 605 (1971).

[9] Cox, A. N., "Allen's astrophysical quantities", 4th ed. Publisher: New York: AIP Press; Springer, 2000. Editedy by Arthur N. Cox. ISBN: 0387987460 (2000).

[10] Lucy, L.B., Gravity-Darkening for Stars with Convective Envelopes, Zeitschrift für Astrophysik, 65, 89 (1967).

[11] Irwin, J.B., Standard Light-Time Curves, Astronomical Journal, 64, 149 (1959).

[12] Kwee, K.K., Investigation of Variations in The Period of Sixteen Bright ShortPeriod Eclipsing Binary Stars, Bulletin of the Astronomical Institutes of the Netherlands, 14: 131(1958).

[13] Applegate, J.H., A Mechanism for Orbital Period Modulation in Close Binaries, Astrophysical Journal, 385, 621(1992). 